\title{
A narrative review of exosomes in vascular calcification
}

\author{
Zheng Qin ${ }^{1}$, Ruoxi Liao ${ }^{1}$, Yuqin Xiong ${ }^{1}$, Luojia Jiang ${ }^{1}$, Jiameng Li ${ }^{1}$, Liya Wang ${ }^{1}$, Mei Han ${ }^{1}$, Si Sun ${ }^{1}$, \\ Jiwen Geng ${ }^{1}$, Qinbo Yang ${ }^{1}$, Zhuyun Zhang ${ }^{1}$, Yupei Li ${ }^{1,2}$, Heyue Dü ${ }^{1}$, Baihai Su${ }^{1}$
}

${ }^{1}$ Department of nephrology and National Clinical Research Center for Geriatrics, West China Hospital of Sichuan University, Chengdu, China; ${ }^{2}$ Institute for Disaster Management and Reconstruction, Sichuan University, Chengdu, China

Contributions: (I) Conception and design: All authors; (II) Administrative support: None; (III) Provision of study materials or patients: None; (IV) Collection and assembly of data: All authors; (V) Data analysis and interpretation: All authors; (VI) Manuscript writing: All authors; (VII) Final approval of manuscript: All authors.

Correspondence to: Prof. Baihai Su. Department of nephrology and National Clinical Research Center for Geriatrics, West China Hospital, Sichuan University, No. 37, Guoxue Lane, Wuhou District, Chengdu 610041, China. Email: imsbh@163.com.

\begin{abstract}
Vascular calcification (VC) is the abnormal deposition of calcium, phosphorus, and other minerals in the vessel wall and can be commonly observed in diabetes, chronic kidney disease, and chronic inflammatory disease. It is closely associated with mortality from cardiovascular events. Traditionally, calcification is considered as a degenerative disease associated with the aging process, while increasing evidence has shown that the occurrence and development of calcification is an active biological process, which is highly regulated by multiple factors. The molecular mechanisms of VC have not yet been fully elucidated. Exosomes, as important transporters of substance transport and intercellular communication, have been shown to participate in VC. The regulation of VC by exosomes involves a number of complex biological processes, which occur through a variety of interaction mechanisms. However, the specific role and mechanism of exosomes in the process of $\mathrm{VC}$ are still not fully understood and require further study. This review will briefly describe the roles of exosomes in the process of VC including in the promotion of extracellular mineral deposits, induction of phenotypic conversion of vascular smooth muscle cells (VSMCs), transport of microRNA between cells, and regulation on autophagy and oxidative stress, with the aim of providing novel ideas for the clinical diagnosis and treatment of VC.
\end{abstract}

Keywords: Vascular calcification (VC); exosome; microRNA

Submitted Nov 08, 2020. Accepted for publication Feb 04, 2021.

doi: $10.21037 /$ atm-20-7355

View this article at: http://dx.doi.org/10.21037/atm-20-7355

\section{Introduction}

Vascular calcification (VC) is the abnormal deposition of calcium, phosphorus, and other minerals in the vessel wall in the form of hydroxyapatite. VC typically occurs in the intima or medial membrane of the aorta, aortic valve, and coronary artery (1). It is often observed in patients with chronic kidney disease (CKD), diabetes, and valvular disease, among others, and is an independent risk factor for cardiovascular events, especially in CKD patients (2-5). Over $60 \%$ of elderly people have calcium salt deposits in the vascular walls, and VC is closely associated with mortality from cardiovascular diseases in the aged population (6).
Sodium thiosulfate and SNF472 are potential available treatments for VC $(7,8)$. In a small randomized controlled study, sodium thiosulfate failed to hinder the progress of abdominal aortic calcification progress, but it positively affected calcification progress in iliac arteries and heart valves, along with several other cardiovascular functional parameters (9). Large-scale randomized controlled trials are necessary to contribute to support the use of and to gain recognition for sodium thiosulfate (10). Raggi et al. found that SNF472 can significantly attenuated the progression of coronary artery calcium and aortic valve calcification in patients with end-stage kidney disease receiving hemodialysis in addition to standard care (8). However, 
further studies are needed to determine the effects of SNF472 on cardiovascular events. There is currently no validated effective treatment with valid evidence for $\mathrm{VC}$, and the underlying mechanisms have not yet been fully elucidated.

Traditionally, calcification is considered as a degenerative disease associated with the aging process. However, increasing evidence has demonstrated that the occurrence and development of calcification is an active and highly regulated complex biological process, which is regulated by multiple factors, such as phenotypic conversion of VSMCs, metabolic homeostasis of calcium and phosphate, inflammation, oxidative stress, autophagy, and extracellular vesicle (EVs) release, among others (11-14). Of these process, osteoblastic phenotypic conversion of VSMCs is a key event in the process of $\mathrm{VC}$, and is often accompanied by upregulation of transcription factors (TFs), such as runtrelated transcription factor 2 (Runx2), muscle segment homeobox (Msx 2), osteopontin (OPN), bone morphogenetic protein-2 (BMP-2), and alkaline phosphatase (ALP). These TFs regulate bone and cartilage formation and the loss of smooth muscle (SM)-specific cell markers proteins such as $\alpha$-smooth muscle actin ( $\alpha$-SMA), smooth muscle myosin heavy chain (SMMHC), h1-calponin, SM22 $\alpha$, and smoothelin (15). When upregulated, these TFs lead to an increase in calcium deposits both intracellularly and extracellularly, which eventually leads to calcification of cells and blood vessels. Further, increased serum calcium and phosphate levels are also associated with VC. A study found that serum inorganic phosphate levels in CKD patients were 1.5-2 times higher than those of the normal population, and the incidence of $\mathrm{VC}$ in $\mathrm{CKD}$ patients is 2-5 times higher than that in the normal population (16). In vitro studies have shown that increased $\mathrm{Ca} 2+$ concentration can reduce calcium-sensing receptor (CaSR, a G-protein coupled receptor related to VC) expression and increase mineral deposition of VSMC (17). In addition, chronic inflammation can induce $\mathrm{VC}$ through cytokines, including interleukin-6 (IL-6), interleukin-1 $\beta$ (IL-1 $\beta$ ) and tumor necrosis factor- $\alpha$ (TNF- $\alpha$ ), via JAK/ STAT3, AMPK, cAMP and NF-kB pathways, among others (18-21). Oxidative stress can promote VC through advanced oxidation protein products (AOPPs) via the AKT, ERK, MAPK, and other pathways $(22,23)$. Another contributing factor of $\mathrm{VC}$ is accelerated senescence of autophagy deficient VSMCs (24). The molecular mechanism of VC requires further research, as it is of great significance in the clinical treatment of VC.

Exosomes, as important intercellular message transporters, have recently been shown to participate in VC $(14,25,26)$. Exosomes are a kind of EVs which are released into the extracellular matrix (ECM) through the fusion of intracellular multivesicular bodies (MVBs) with cell membrane diameters of 30-100 nm (27). A variety of functions affecting the ECM involve EVs, including matrix degradation, cross-linking of matrix proteins, and matrix calcification (28). Due to the variety and abundance of specific cargos including proteins, lipids, and nucleic acids, exosomes have emerged as regulators of cell-tocell communication during different physiological and pathological processes, including cell proliferation, differentiation, apoptosis, and migration (29,30). Kapustin et al. found exosomes in the arteries of CKD patients and observed that CD63, a biomarker of exosomes, was colocated with calcification, suggesting that exosomes are involved in the process of $\mathrm{VC}$ regulation (26). However, the specific role and mechanism of exosomes in the process of $\mathrm{VC}$ are still not fully understood and need to be investigated further.

This review article discusses the formation and characteristics of exosomes and their potential mechanisms in $\mathrm{VC}$. We present the following article in accordance with the Narrative Review reporting checklist (available at http:// dx.doi.org/10.21037/atm-20-7355).

\section{Methods}

We conducted a literature search for papers published up to October 2020 on the topic of exosomes in VC using the PubMed, MEDLINE and Embase databases with following key words: "exosome", "exosomal", "extracellular vesicles" or "microvesicles" plus "vascular calcification", "calcification", "osteogenetic", "osteoblastic" or "osteogenesis", and the retrieved articles were further analyzed.

\section{Discussion}

\section{Formation of exosomes and their characteristics}

Exosomes are a type of typical EVs with a diameter of about $30-100 \mathrm{~nm}$. They are secreted by cells with lipid bilayer membrane structure and contain cargos such as proteins, lipids, and nucleic acids, etc., which can be widely found in body fluids (27). Essentially, exosomes are MVBs that are released to the extracellular surface after fusion with the cytoplasmic membrane. The process of exosome formation 
is as follows: First, the plasma membrane depresses inward to form the endosome. Then, the endosomal-limiting membrane reoccurs in several places to form intraluminal vesicles (ILVs), which further form mature MVBs. Eventually, when the MVBs have fused with the cell surface, ILVs are released into the extracellular domain, that is, exosomes (31).

Exosomes usually express CD63, CD81, CD9, and other specific markers, which are often used for their identification. Different types of cells release exosomes into body fluids and various microenvironments. Exosomes mediate transcription products and lipids with various functions to target cells through the humoral circulation and play a role in nearby or distant cells, thus regulating their molecular and phenotypic changes. At present, there are believed to be 3 main steps of exosomes uptake by target cells. First, exosomes are endocytized or internalized into the endocytic compartment, and their contents are released into the cytoplasm of the recipient cell through reverse fusion. After that, exosomes can be fused with the endosomal membrane or be degraded by targeted lysosomes. Then, receptor-ligand interaction. Finally, the exosome membrane fuses with the cytoplasmic membrane, and the contents are released directly into the cytoplasm of the recipient cell (32).

Numerous studies have shown that exosomes share many characteristics of matrix vesicles (MVs), which are unique extracellular membrane-bound microparticles that serve as initial sites for mineral formation. Indeed, analyses of size, morphology, and lipid and protein content have indicated that MVs and exosomes are homologous structures, suggesting that exosomes may play a similar role in the induction of mineral deposition during calcification as MVs (33). Both exosomes and MVs are enriched in calcified vascular walls. Kapustin et al. found that VSMCsecreted exosomes could promote the occurrence of $\mathrm{VC}$ through mineral deposition, and the expression of fetuin-A (a known calcification inhibitor) was significantly reduced in secreted exosomes (34). Chen et al. found that in patients with CKD, exosomes secreted by calcified VSMCs delivered special proteins and RNA, through which they played a role in promoting $\mathrm{VC}$ (35). MVs released from endothelial cells exposed to proinflammatory stimuli were found to be enriched in BMP-2, which promoted VSMCs calcification after vesicle phagocytosis (36).

In cardiovascular diseases, exosomes can be secreted by VSMCs, endothelial cells, macrophages, and other cells, and participate in intercellular communication (37). Studies have shown that in the process of coronary artery calcification, microRNA (miRNA) and proteins are transported via exosomes from various cells to VSMC (38). Pan et al. found miRNA profiles were significantly altered in exosomes from VSMCs associated with coronary artery calcification (39). Calcified vascular walls are enriched in exosomes. However, the role of exosomes in the formation of carotid calcified plaque, a type of cardiovascular calcification described as clinically stable that is frequently found in asymptomatic patients with carotid stenosis, has not been extensively studied $(40,41)$. Dolz et al. found that specific circulating miRNAs transported by exosomes, such as miR-199b-3p, miR-27b3p, miR-130a-3p, miR-221-3p, and miR-24-3p, were significantly highly expressed in those patients with asymptomatic carotid artery stenosis progression (42). The characteristics of exosomal transport of functional contents to target cells make exosomes not only an indicator of disease diagnosis and prognosis, but also important carriers in targeted therapy $(30,37)$. Melatonin can alleviate VC and ageing through exosomal miR-204/miR-211 cluster in a paracrine manner through exosomes (43). However, considering that there might be double cerebrovascular symptomatic peaks according to calcium scores in calcified carotid plaques (44), reducing calcification through exosomes and other means may increase the risk of developing calcification symptoms, and exosomal calcification reduction may not necessarily be appropriate for all patients with VC.

\section{The role of exosomes in $V C$}

Phenotypic conversion of VSMCs is a key event in the process of $\mathrm{VC}$, in which exosomes have been proven to play an important role. Under normal physiological conditions, VSMCs exhibit a contractile phenotype and regulate microenvironmental homeostasis by actively releasing exosomes that contain endogenous calcification inhibitors, such as fetuin A, matrix Gla protein (MGP), osteoprotegerin (OPG), and bone morpho-genetic protein-7 (BMP-7), so as to inhibit the occurrence of VC (45). However, under the stimulus of pathological factors, such as mechanical injury, oxidative stress, apoptosis and phosphate metabolism disorder and other factors, VSMCs turn into synthetic phenotypes and related factors of osteogenic differentiation are activated, such as OPN, osteocalcin (OCN), bone morphogenetic protein-2 (BMP-2), ALP, etc. As a result, the transformation of cells into osteoblastic/chondroid phenotypes with presenting features related to osteoblasts is promoted. The release of calcified exosomes increases with 
the depletion of MGP and fetuin-A, and enrichment with a protein-lipid complex consisting of phosphatidyl serine (PS) and annexin. The protein-lipid complex converts exosomes to the nidus for calcification by providing mineral nucleation sites. Mineral deposition and calcification are formed through the interaction of calcified exosomes with elastic proteins and collagen fibers in the ECM, which promotes the occurrence of $\mathrm{VC}$.

Studies have shown that exosomes participate in processes including the initiation of mineral deposition, promotion of phenotypic conversion of VSMC, mediation of miRNA transport, autophagy and oxidative stress reaction, and so on, so as to regulate the process of $\mathrm{VC}$.

\section{Exosomes promote extracellular mineral deposits to induce VC}

Many studies have shown that exosomes can participate in VC formation by acting as mineralization sites for minerals such as calcium and phosphorus (34). Typically, $\mathrm{VC}$ is characterized by ectopic deposits of minerals, and MVs is regarded as a major participant in the calcification process. The release of MVs is generally considered to be an adaptive response. Normally, MVs expel excess calcium from the cell, thus providing protection against intracellular calcium overload. However, with the passage of time and the loss of calcification inhibitors, MVs are transformed into calcified MVs with the loss of calcification inhibitors, which promotes the formation of calcification foci (46). Numerous studies have shown that exosomes share many characteristics of MVs and may have a similar role in the induction of mineral deposition during calcification as initial sites for mineral formation.

Studies have shown that the first calcification lesion formed by exosomes is located in proximity to collagen fibers and elastin. They were found to differ in size and mineral content, and seemed to arise from VSMCs in mineralized sites, as well as VSMCs, macrophages, endothelial cells, and platelets in atherosclerotic plaques $(47,48)$. High-phosphate and calcium levels can stimulate the excretion of VSMC exosomes. Sphingomyelin phosphodiesterase 3 (SMPD3) is a regulatory factor involved in exosomes formation. An in vitro study found that the expression of SMPD3 was increased when cells were exposed to specific conditions such as high phosphorus and high calcium levels $(3,49)$. Exosomes secretion was further promoted through the conversion of sphingolipin to ceramide and the induction of binding of clathrin-coated microregions by SMPD3 (50). In vitro studies have confirmed that inhibition of SMPD3 hinders the production of exosomes and calcification formation during induced VC (34). These specific exosomes obtain mineral compounds and may further initiate or aggravate the mineral deposition process. When intracellular Ca2+ content is too high, exosomes can absorb calcium and phosphorus and other mineral complexes to maintain the homeostasis of intracellular mineral metabolism, which results in their calcified (51). The secreted content of MGP in these calcified exosomes secreted during $\mathrm{VC}$ is low, whereas the hydroxyapatite content is high, which is also the initiating factor that leads to mineral deposition in the formation of microcalcification (26). The low concentrations of fetuin-A and Gla rich protein (GRP) in calcified exosomes causes them to aggregate in the collagen-sparse region to form microcalcifications upon release into the ECM (52). In addition to minerals in exosomes, external PS can also bind to calcium ion binding proteins, such as annexin A2, A5, and A6, thus forming hydroxyapatite deposition in the inner or outer part of the exosome membrane; thus, the exosomes transform into primary foci for calcification by providing mineral nucleation sites $(48,53)$. Studies have also shown that annexin can also be released into the ECM through exosomes, suggesting that exosomes can enhance calcification by promoting the binding of PS to calcium-binding proteins (54). Ciceri et al. found that iron citrate could reduce VC induced by high phosphate level through the prevention of exosome osteo-chondrogenic shift by reducing phosphate load, suggesting that free phosphate in vitro is an essential factor for calcification in vitro (49). In addition to the extracellular environment of high inorganic phosphorus, phosphatases on the surface of exosomes can convert pyrophosphate into inorganic phosphorus and further increase the concentration of free inorganic phosphorus outside the cells, thus promoting the calcification process and forming positive feedback (55) (Figure 1).

In summary, exosomes promote extracellular mineral deposits to induce $\mathrm{VC}$, and the exosome secretion is activated in the background of VC. Thus, interference with exosome secretion can be used as a new target to prevent VC.

\section{Exosomes regulate VC through the phenotypic conversion of VSMCs}

Phenotypic conversion of VSMCs from a contractile to a synthetic state contributes to vascular pathologies including VC, restenosis, and atherosclerosis (56). Phenotypic conversion of VSMCs is a key link in the 


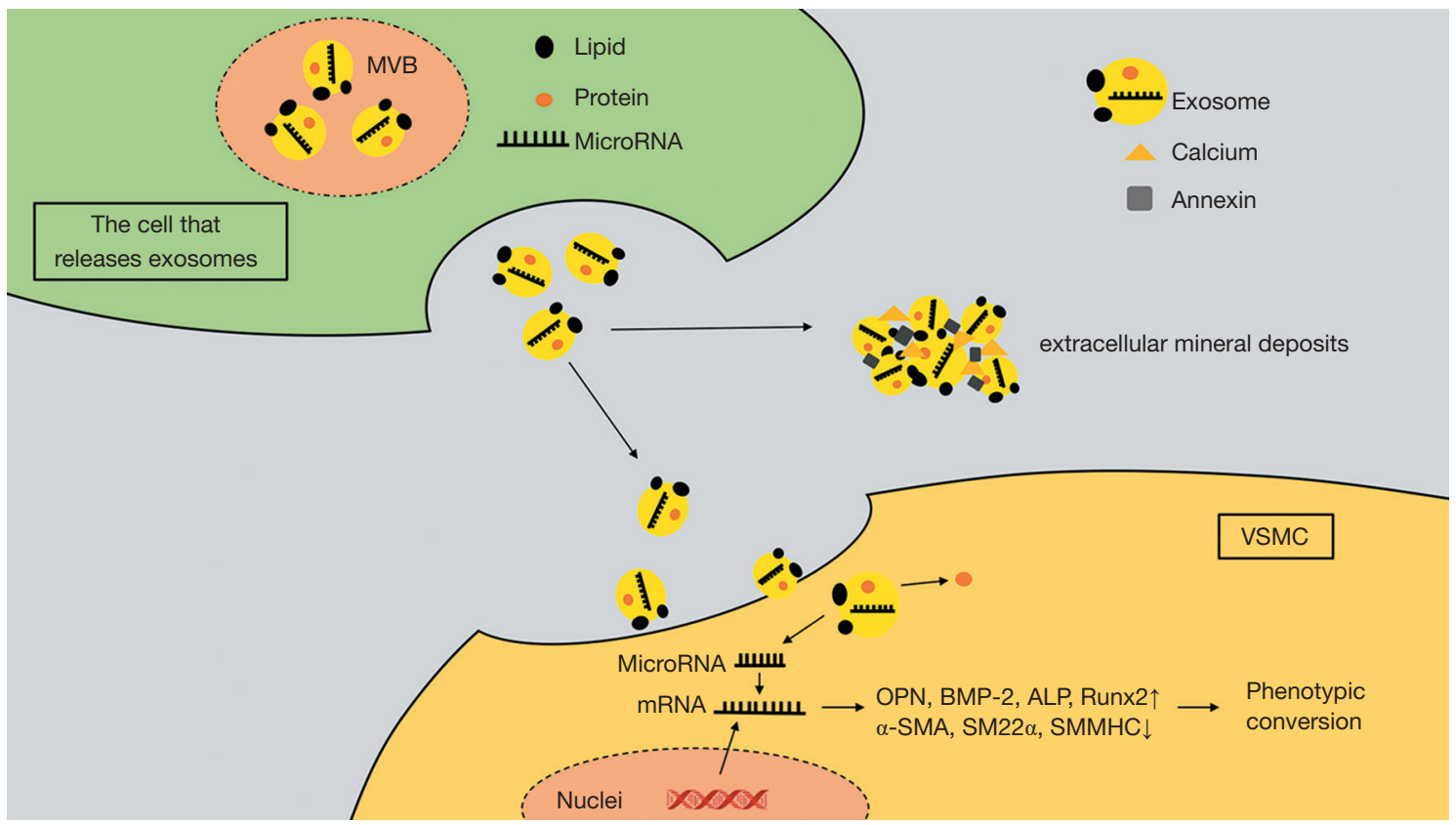

Figure 1 Exosomes promote extracellular mineral deposits and transport microRNA between cells to induce VC. VC, vascular calcification.

development of VC. Histological analysis of calcified human blood vessels revealed that OPN, Cbfa1, Runx2, and other markers related to osteogenic differentiation were significantly highly expressed, whereas the levels of contraction markers including $\alpha$-SMA, SM $22 \alpha$, and SMMHC were decreased $(57,58)$. Further, in the process of atherosclerosis and endothelial cell injury, inflammatory factors and hemodynamic changes stimulate the smooth muscle cells (SMCs) to differentiate from stable phenotype to synthetic phenotype, giving them stronger ability to migrate and proliferate, thus facilitating their migration through the internal elastic membrane into the lining layer and promoting plaques formation (59). The results of highresolution micro-computed tomography also showed that there were calcified exosomes in the microcalcification foci of the fibrous cap of atherosclerotic plaque (60). In their in vitro experiment, Pan et al. found that exosomes from calcified mouse VSMCs could induce significant calcification of un-calcified mouse VSMCs (39). Li et al. also found that high-glucose-stimulated human umbilical vein endothelial cells (HUVECs) could secret exosomes which induced calcification/senescence in VSMCs (61). These results suggest that exosomes are involved in regulating the formation and development of $\mathrm{VC}$, and may be closely related to osteoblastic phenotypic transformation of VSMCs.

During the phenotypic conversion of VSMCs, exosomes can be used as unique signal transport carriers to participate in the regulation of cell proliferation, migration, apoptosis, calcification, and differentiation (62). Sortilin is a key transport factor that regulates VSMCs calcification. It is commonly found in calcified human blood vessels and atherosclerotic plaques in mice. By regulating the load of MVs on tissue non-specific alkaline phosphatase (TNAP) associated with calcification protein, sortilin can be transformed into a vesicle with calcification potential and can participate in the formation of microcalcification (63). Calcified MVs contain dysfunctional miRNAs that induce gene expression of osteogenic markers such as Runx2, TNAP, Smad1, Osterix, and proinflammatory factors (64). Expression of the phenotypic markers of VSMC contraction is usually negatively correlated with exosome secretion. Platelet-derived growth factor BB (PDGF-BB) promotes exosome secretion, and reduces the representation of the phenotypic markers of SMC contraction. Milk fat globuleepidermal growth factor 8 (MFG-E8) is a precursor of amyloid protein, which is an age-related protein and that is formed in the aortic membrane of aged blood vessels. This factor can induce osteoblastic phenotype of VSMCs through the ERK signaling pathway and promote 


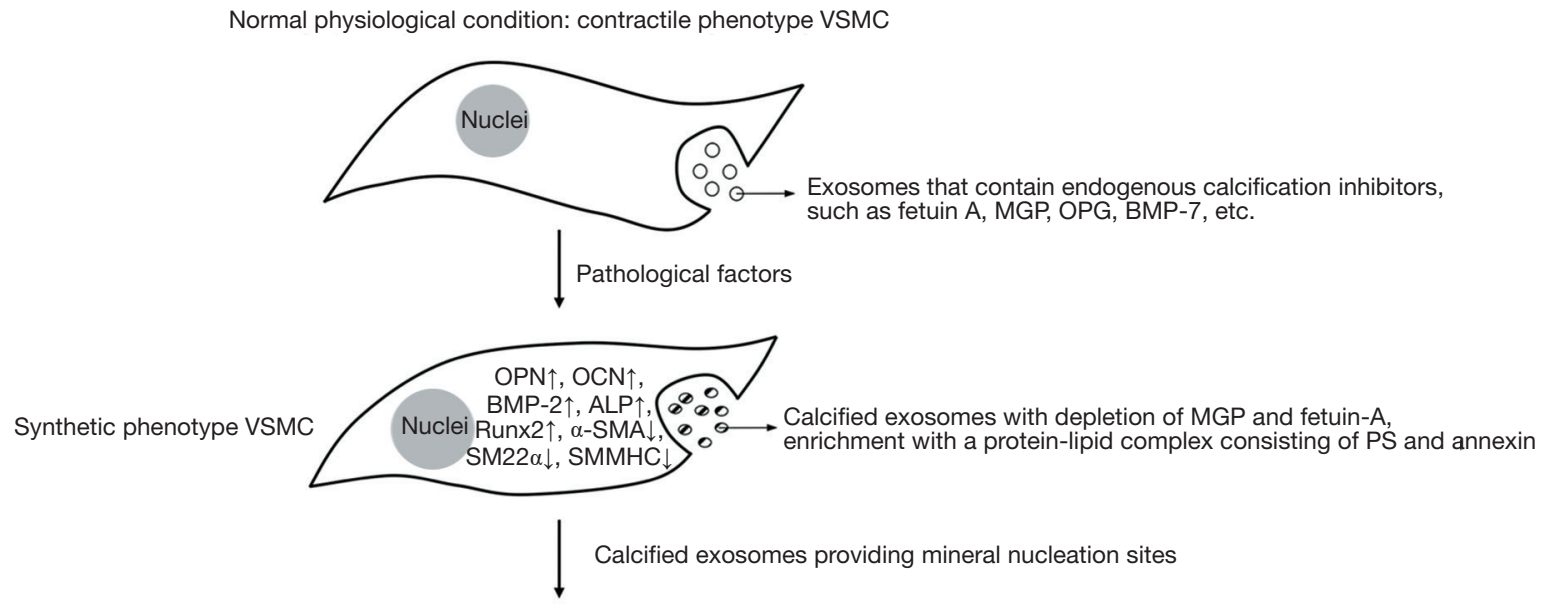

ECM

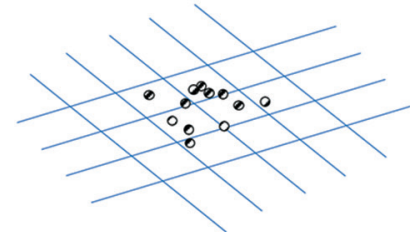

Mineral deposition and calcification are formed through the interaction of calcified exosomes with ECM

Figure 2 Exosomes regulate VC through the phenotypic conversion of VSMCs. VC, vascular calcification; SMCs, vascular smooth muscle cells.

calcification (65), and studies have found that MFG-E8rich exosomes released by VSMCs increase with age. Thus, exosomes may participate in VC by inducing VSMC phenotypic conversion (Figure 2).

\section{Exosomal miRNAs regulate VC}

The role of exosomes in intercellular communication has been widely recognized. Exosomes cargos include RNA, cytokines, proteins, and lipids. Studies have shown that the components of exosomes cargos differ significantly according to the cells that the exosomes origin. At present, an increasing number of researchers believe that exosomes play an important role in the intercellular communication during the process of $\mathrm{VC}$ (66). A large number of studies have focused on the role of exosomes in inducing mineral deposition during $\mathrm{VC}$, but the role of exosomes in information transfer in $\mathrm{VC}$ has not yet been clarified.

MiRNAs are an important class of endogenous, single stranded, non-coding RNAs, which are involved in the regulation of gene expression and translation (67). The expression of miRNAs can be specific to different tissues and cell types, and miRNAs play an important role in a variety of biological processes, including inflammation, tumor, cardiovascular disease, etc. (67). MiRNAs suppress gene expression through imperfect base pairing to the 3' untranslated region of target mRNAs, leading to repression of protein production or mRNA degradation. Importantly, a single miRNA may affect the transcription of multiple genes involved in common pathways. During VC, upregulated miRNAs include miRNA-221 (68), miRNA-222 (68), miRNA-762 (69), miRNA-714 (69), miRNA-712 (69), and miRNA-210 (70). Conversely, several miRNAs involved in calcification inhibition are downregulated during $\mathrm{VC}$, including miRNA-26 (71), miRNA-30 (72,73), miRNA125b (74,75), and miRNA-204 (76).

Exosomes are one of the carriers of extracellular miRNA, which can protect miRNAs from degradation. The miRNAs encapsulated in exosomes have been shown to be non-identical to the maternal cells, suggesting the selectivity of this process. Exosomal loaded miRNAs are transported from releasing cells to receptor target cells, thereby carrying out gene regulation functions on receptor target cells. Huang et al. found that the selective enrichment of miRNAs in exosomes was determined by changes in exosome-secreting cells (77). Further, the types of exosometransporting miRNAs vary according to the environment; these miRNAs are involved in the regulation of different genes and produce specific biological effects, such as cell phenotypic changes and inflammatory responses, through the transportation of miRNAs $(78,79)$.

Exosomes from different sources can participate in the regulation of $\mathrm{VC}$ by transporting miRNAs to recipient 
VSMCs (69). Cui et al. found that exosomes released by mineralized osteoblasts $\mathrm{Mc} 3 \mathrm{t} 3$-e 1 contribute to the osteogenic differentiation of ST2 cells via a complex network of exosomal miRNAs (80). Guo et al. found that bone marrow mesenchymal stem cell (MSC)derived exosomes can alleviate high phosphorusinduced calcification in human aortic VSMCs through the modification of miRNA profiles (81). Kapustin et al. found that exosomes derived from VSMCs are rich in miRNA-143 and proteins regulating cell adhesion and migration, which can participate in the regulation of cell proliferation and migration through autocrine and paracrine manners (26). An in vivo study showed that exosomes derived from melatonin-treated VSMCs could reduce VC and aging in 5/6-nephrectomy plus high-phosphate diet-treated mice, while these effects were largely abolished by inhibition of exosomal miR-204 or miR-211 (43).

The underlying mechanisms by which exosomes affect VC via transporting miRNA are still not fully understood and may vary among different conditions. Changes of miRNAs in exosomes can regulate osteogenic differentiation of cells by promoting the expression of Runx 2 and activating related signaling pathways, for example, the $\mathrm{Wnt} / \beta$-catenin pathways. Studies have shown that miRNAs with increased expression in the $\mathrm{VC}$ process can promote the osteogenic transformation of SMCs by targeting anti-calcification proteins or contractility markers, while miRNAs with decreased expression can inhibit the osteogenic transformation of SMCs by targeting osteogenic TFs (25). It has been shown that miRNA-233 transforms SMCs from contractile phenotype to synthetic phenotype and promotes VC (82). Other miRNAs with similar effects include miRNA-712, miRNA-714, and miRNA-762 (83). In contrast, miRNA-26a inhibits the differentiation of SMCs, and miRNAs that regulate similar effects of phenotypic transformation by targeting SMAD1 and SMAD4 include miRNA-29a/b and miRNA-125b $(83,84)$.

Many studies have shown that the interaction between endothelial cells and SMCs plays a key role in the maintenance of vascular wall homeostasis, and exosomes are important carriers to realize cell-cell interaction and information transfer under both normal and pathological conditions. On the one hand, SMCs release secreted bodies which regulate endothelial cell adhesion, migration, and autophagy. X-box binding protein 1 (XPB1) is an important binding protein that regulates cell function. An in vitro experiment showed that its expression in endothelial cells were regulated by VSMCs, mainly through the VSMC-originated MVs containing miRNA-150 and miRNA-150, which regulate endothelial cell migration via the VEGF-A/VEGFR/PI3K/Akt signaling pathway (85). In another in vitro experiment, Lombardo et al. found that miRNA-22/222 originating from human aortic SMCs exosomes can inhibit autophagy of HUVECs through the PTEN/Akt signaling pathway. On the other hand, miRNAs in endothelial cells exosomes can regulate VSMCs gene expression and phenotypic conversion (86). Hergenreider et al. found that under the effect of fluid shear stress, endothelial cells released exosomes rich in miRNA-143/145 and could be absorbed by VSMCs; in turn, the phenotypic conversion of VSMCs was inhibited, and thus, the occurrence of VC was prevented (87) (Figure 1).

\section{Other mechanisms}

Intercellular crosstalk through exosomes is an important mechanism for calcification in $\mathrm{VC}$. VC is promoted by the transport of exosome cargos, such as miRNAs and proteins between cells. Studies have found that exosomal Notch3 and versican from high glucose-stimulated endothelial cells regulates VSMCs calcification/senescence and may lead to diabetes-related VC $(61,88)$. Further, several studies have shown that exosome-mediated intercellular crosstalk is dependent on heparin sulphate proteoglycan (HSPG), which protects VSMCs from various toxic substances and circulating inflammatory cells, thus preventing VC (89). The decreased expression of HSPG in exosomes was found to expose HSPG on the cell surface, which further mediated the internalization of BMP2 and promoted the osteogenic phenotypic conversion (90). In addition, inhibition of HSPG expression on the cell surface results in reduced efficiency of exosome uptake, which may can be a potential mechanism to reduce calcification.

Autophagy is a key regulator of cell metabolism and homeostasis and plays an important role in maintaining normal vascular cell function. In recent years, studies have shown that appropriate enhancement of autophagy can inhibit exosome secretion to form mineral nucleation sites, thereby reducing VSMC calcification (91). Autophagy accelerates the degradation of exosomes and reduces the secretion of exosomes, which is mediated by autophagosomal lysosome fusion. Further, autophagy appears to interfere with miRNA loading into exosomes in VSMCs, which may subsequently interfere with mineral deposition and osteogenic phenotypic conversion (92). A series of studies have focused on the role of exosomes in the relationship between autophagy and VC. Dai et al. 
reported that autophagy may be an endogenous protective mechanism for combatting $\mathrm{VC}$ induced by high phosphate level by reducing the release of calcified vesicles (93). They found that the autophagy inhibitor 3-methyladenine (3-MA) could significantly promote the release of calcified exosomes induced by elevated phosphorus, enhance the activity of ALP, and increase the calcium deposition in SMCs, while the autophagy inducer valproic acid could inhibit the release of calcified exosomes induced by high phosphate level.

Intracellular calcium overload leads to a series of disorders in superoxide metabolism, resulting in oxidative stress (23). Oxidative stress has been revealed as one of the important mechanisms leading to the osteogenic phenotype conversion of VSMCs in relation to $\mathrm{VC}$, by excessive production of reactive oxygen species (ROS) (22). ROS may cause lipid peroxidation, as well as serious damage to DNA and proteins, and other abnormal biochemical changes (94). Studies found that the level of oxidative stress in patients with CKD is closely related to cardiovascular disease and VC $(95,96)$. Advanced glycation end-products (AGEs) activate the receptors of advanced glycation end-products (RAGEs) to initiate oxidative stress, an important mechanism for ROS production in diabetics. In diabetics, AGEs upregulate ROS production through RAGEs, increasing ALP activity, and promoting VC (97). Wei et al. found that the activation of the KEAP1/NRF2/P62 signaling pathway can reduce the high-phosphate induced VSMCs by inhibiting the production of ROS (98). Several studies have suggested that exosomes are associated with oxidative stress in VC. Patel et al. found that exosomes from breast cancer cells can promote the production of ROS in the recipient primary breast epithelial cells, which indicates that exosomes may regulate the ROS production to get involved in VC (99). Liu et al. observed that the decreased expression of miR-210 during the progression of $\mathrm{VC}$ and miR-210 in exosomes can reduce ROS production in recipient endothelial cells (100). Research has shown that the expression of miR-30 decreases after pro-calcification stimulation, while miR-30 could further reduce the oxidative stress levels by regulating AGE/ RAGE interaction $(101,102)$. Meanwhile, exosomal transfer of pro-angiogenic miRNAs, including miRNA-30, plays a key role in MSC-mediated angiogenesis and stem cell-toendothelial cell communication (103). Under the stimulation of AGEs, MSC-secreted exosomes containing miRNA-146a, along with exosomes with high expression of miRNA-164a were transported to VSMCs, thus, inhibiting the calcification induced by AGE-bovine serum albumin (AGE-BSA) (104). After induction of replicative senescence of VSMCs and calcification were observed, the release of exosomes by VSMCs was increased, and genes highly expressed in osteoblasts, such as ALP, ColI and Runx2, were significantly upregulated in the senescent VSMCs, suggesting their osteoblastic transition during the senescence, which may be regulated by the AMPK/ TSC2/mTOR/S6K1 signaling pathway $(105,106)$. The studies above indicate that exosomes may modulate oxidative stress by regulating the production of ROS, and then participate in the regulation of VC.

\section{Conclusions}

Commonly presenting in diabetes, calcific aortic valve disease, CKD, and chronic inflammatory disease, $\mathrm{VC}$ substantially increases the incidence and mortality of cardiovascular events. In this review, we discussed the roles of exosomes in VC. Exosomes can regulate VC through promoting the formation of extracellular mineral deposits, inducing phenotypic conversion of VSMCs, transporting microRNA between cells, regulating autophagy and oxidative stress, and other activities. Further research on the characteristics of exosomes and their role in VC is needed and expected to provide novel ideas and targets for the clinical diagnosis and treatment of VC.

\section{Acknowledgments}

Funding: This work was supported by the State Key Research Programme of China (Grant No. 2016YFC1103004, Grant No. 2016YFC1103003), the Key Project of Research and Development of Science and Technology Department of Sichuan Province (No. 2018FZ0102), the Science and technology achievement transformation fund of West China Hospital of Sichuan University (No. CGZH19006), and the world-class university construction fund of Sichuan university (No. 2040204401012), National Clinical Research Center for Geriatrics, West China Hospital, Sichuan University (Grant No. Z2018B10).

\section{Footnote}

Reporting Checklist: The authors have completed the Narrative Review reporting checklist. Available at http:// dx.doi.org/10.21037/atm-20-7355

Peer Review File: Available at http://dx.doi.org/10.21037/ atm-20-7355 
Conflicts of Interest: All authors have completed the ICMJE uniform disclosure form (available at http://dx.doi. org/10.21037/atm-20-7355). BS serves as an unpaid editorial board member of Annals of Translational Medicine from Sep 2020 to Aug 2021. The authors have no other conflicts of interest to declare.

Ethical Statement: The authors are accountable for all aspects of the work in ensuring that questions related to the accuracy or integrity of any part of the work are appropriately investigated and resolved.

Open Access Statement: This is an Open Access article distributed in accordance with the Creative Commons Attribution-NonCommercial-NoDerivs 4.0 International License (CC BY-NC-ND 4.0), which permits the noncommercial replication and distribution of the article with the strict proviso that no changes or edits are made and the original work is properly cited (including links to both the formal publication through the relevant DOI and the license). See: https://creativecommons.org/licenses/by-nc-nd/4.0/.

\section{References}

1. Demer LL, Tintut Y. Vascular calcification - Pathobiology of a multifaceted disease. Circulation 2008;117:2938-48.

2. Geisel MH, Bauer M, Hennig F, et al. Comparison of coronary artery calcification, carotid intima-media thickness and ankle-brachial index for predicting 10-year incident cardiovascular events in the general population. Eur Heart J 2017;38:1815-22.

3. Kapustin AN, Shanahan CM. Emerging roles for vascular smooth muscle cell exosomes in calcification and coagulation. J Physiol 2016;594:2905-14.

4. Wu M, Rementer C, Giachelli CM. Vascular Calcification: An Update on Mechanisms and Challenges in Treatment. Calcif Tissue Int 2013;93:365-73.

5. Zhang $\mathrm{K}$, Zhang $\mathrm{Y}$, Feng $\mathrm{W}$, et al. Interleukin-18 Enhances Vascular Calcification and Osteogenic Differentiation of Vascular Smooth Muscle Cells Through TRPM7 Activation. Arterioscler Thromb Vasc Biol 2017;37:1933-43.

6. Hsu JJ, Lim J, Tintut Y, et al. Cell-matrix mechanics and pattern formation in inflammatory cardiovascular calcification. Heart 2016;102:1710-5.

7. O'Neill WC. Sodium Thiosulfate: Mythical Treatment for a Mysterious Disease? Clin J Am Soc Nephrol 2013;8:1068-9.
8. Raggi P, Bellasi A, Bushinsky D, et al. Slowing Progression of Cardiovascular Calcification With SNF472 in Patients on Hemodialysis Results of a Randomized Phase 2b Study. Circulation 2020;141:728-39.

9. Djuric P, Dimkovic N, Schlieper G, et al. Sodium thiosulphate and progression of vascular calcification in end-stage renal disease patients: a double-blind, randomized, placebo-controlled study. Nephrol Dial Transplant 2020;35:162-9.

10. Piccoli GB, Torreggiani M, Gendrot L, et al. Setting the clock back: new hope for dialysis patients. Sodium thiosulphate and the regression of vascular calcifications. J Nephrol 2021;34:23-5.

11. Bostroem KI, Yao J, Guihard PJ, et al. Endothelialmesenchymal transition in atherosclerotic lesion calcification. Atherosclerosis 2016;253:124-7.

12. Libby P, Ridker PM, Hansson GK. Progress and challenges in translating the biology of atherosclerosis. Nature 2011;473:317-25.

13. Rogers MA, Aikawa E. Cardiovascular calcification: artificial intelligence and big data accelerate mechanistic discovery. Nat Rev Cardiol 2019;16:261-74.

14. Yang W, Zou B, Hou Y, et al. Extracellular vesicles in vascular calcification. Clin Chim Acta 2019;499:118-22.

15. Balogh E, Toth A, Mehes G, et al. Hypoxia Triggers Osteochondrogenic Differentiation of Vascular Smooth Muscle Cells in an HIF-1 (Hypoxia-Inducible Factor 1)-Dependent and Reactive Oxygen Species-Dependent Manner. Arterioscler Thromb Vasc Biol 2019;39:1088-99.

16. Braun J, Oldendorf M, Moshage W, et al. Electron beam computed tomography in the evaluation of cardiac calcification in chronic dialysis patients. Am J Kidney Dis 1996;27:394-401.

17. Alam M, Kirton JP, Wilkinson FL, et al. Calcification is associated with loss of functional calcium-sensing receptor in vascular smooth muscle cells. Cardiovasc Res 2009;81:260-8.

18. Navarro-Gonzalez JF, Mora-Fernandez C, Muros M, et al. Mineral Metabolism and Inflammation in Chronic Kidney Disease Patients: A Cross-Sectional Study. Clin J Am Soc Nephrol 2009;4:1646-54.

19. Shao JS, Cheng SL, Sadhu J, et al. Inflammation and the Osteogenic Regulation of Vascular Calcification A Review and Perspective. Hypertension 2010;55:579-92.

20. Tintut Y, Patel J, Parhami F, et al. Tumor necrosis factoralpha promotes in vitro calcification of vascular cells via the cAMP pathway. Circulation 2000;102:2636-42.

21. Son BK, Akishita M, Iijima K, et al. Adiponectin 
antagonizes stimulatory effect of tumor necrosis factor-alpha on vascular smooth muscle cell calcification: Regulation of growth arrest-specific gene 6-mediated survival pathway by adenosine 5 '-monophosphate-activated protein kinase. Endocrinology 2008;149:1646-53.

22. Byon CH, Javed A, Dai Q, et al. Oxidative stress induces vascular calcification through modulation of the osteogenic transcription factor Runx2 by AKT signaling. J Biol Chem 2008;283:15319-27.

23. Nguyen NT, Nguyen TT, Da Ly D, et al. Oxidative stress by $\mathrm{Ca} 2+$ overload is critical for phosphate-induced vascular calcification. Am J Physiol Heart Circ Physiol 2020;319:H1302-12.

24. Grootaert MOJ, Moulis M, Roth L, et al. Vascular smooth muscle cell death, autophagy and senescence in atherosclerosis. Cardiovasc Res 2018;114:622-34.

25. Zhang C, Zhang K, Huang FF, et al. Exosomes, the message transporters in vascular calcification. J Cell Mol Med 2018;22:4024-33.

26. Kapustin AN, Chatrou MLL, Drozdov I, et al. Vascular Smooth Muscle Cell Calcification Is Mediated by Regulated Exosome Secretion. Circ Res 2015;116:1312-23.

27. Ibrahim A, Marban E. Exosomes: Fundamental Biology and Roles in Cardiovascular Physiology. Annu Rev Physiol 2016;78:67-83.

28. Lewin S, Hunt S, Lambert DW. Extracellular vesicles and the extracellular matrix: a new paradigm or old news? Biochem Soc Trans 2020;48:2335-45.

29. van Balkom BWM, de Jong OG, Smits M, et al. Endothelial cells require miR-214 to secrete exosomes that suppress senescence and induce angiogenesis in human and mouse endothelial cells. Blood 2013;121:3997-4006.

30. Wang J, Sun X, Zhao J, et al. Exosomes: A Novel Strategy for Treatment and Prevention of Diseases. Front Pharmacol 2017;8:300.

31. van Niel G, D'Angelo G, Raposo G. Shedding light on the cell biology of extracellular vesicles. Nat Rev Mol Cell Biol 2018;19:213-28.

32. Wang Y, Xie Y, Zhang A, et al. Exosomes: An emerging factor in atherosclerosis. Biomed Pharmacother 2019;115:108951.

33. Shapiro IM, Landis WJ, Risbud MV. Matrix vesicles: Are they anchored exosomes? Bone 2015;79:29-36.

34. Kapustin AN, Davies JD, Reynolds JL, et al. Calcium Regulates Key Components of Vascular Smooth Muscle Cell-Derived Matrix Vesicles to Enhance Mineralization. Circ Res 2011;109:e1-12.

35. Chen NX, O'Neill KD, Moe SM. Matrix vesicles induce calcification of recipient vascular smooth muscle cells through multiple signaling pathways. Kidney Int 2018;93:343-54.

36. Buendia P, Montes de Oca A, Madueno JA, et al. Endothelial microparticles mediate inflammation-induced vascular calcification. FASEB J 2015;29:173-81.

37. Boulanger CM, Loyer X, Rautou PE, et al. Extracellular vesicles in coronary artery disease. Nat Rev Cardiol 2017;14:259-72.

38. Tkach M, Thery C. Communication by Extracellular Vesicles: Where We Are and Where We Need to Go. Cell 2016;164:1226-32.

39. Pan W, Liang J, Tang H, et al. Differentially expressed microRNA profiles in exosomes from vascular smooth muscle cells associated with coronary artery calcification. Int J Biochem Cell Biol 2020;118:105645.

40. Nandalur KR, Baskurt E, Hagspiel KD, et al. Carotid artery calcification on CT may independently predict stroke risk. AJR Am J Roentgenol 2006;186:547-52.

41. de Weert TT, Cakir H, Rozie S, et al. Intracranial Internal Carotid Artery Calcifications: Association with Vascular Risk Factors and Ischemic Cerebrovascular Disease. AJNR Am J Neuroradiol 2009;30:177-84.

42. Dolz S, Gorriz D, Ignacio Tembl J, et al. Circulating MicroRNAs as Novel Biomarkers of Stenosis Progression in Asymptomatic Carotid Stenosis. Stroke 2017;48:10-6.

43. Xu F, Zhong JY, Lin X, et al. Melatonin alleviates vascular calcification and ageing through exosomal miR-204/ miR-211 cluster in a paracrine manner. J Pineal Res 2020;68:e12631.

44. Katano H, Mase M, Nishikawa Y, et al. Calcified Carotid Plaques Show Double Symptomatic Peaks According to Agatston Calcium Score. J Stroke Cerebrovasc Dis 2015;24:1341-50.

45. Touyz RM, Montezano AC. Vascular smooth muscle cells sense calcium: a new paradigm in vascular calcification. Cardiovasc Res 2009;81:237-9.

46. Bommanavar S, Hosmani J, Togoo RA, et al. Role of matrix vesicles and crystal ghosts in bio-mineralization. J Bone Miner Metab 2020;38:759-64.

47. Leroyer AS, Isobe H, Leseche G, et al. Cellular origins and thrombogenic activity of microparticles isolated from human atherosclerotic plaques. J Am Coll Cardiol 2007;49:772-7.

48. Bobryshev YV, Killingsworth MC, Huynh TG, et al. Are calcifying matrix vesicles in atherosclerotic lesions of cellular origin? Basic Res Cardiol 2007;102:133-43.

49. Ciceri P, Falleni M, Tosi D, et al. High-phosphate induced 
vascular calcification is reduced by iron citrate through inhibition of extracellular matrix osteo-chondrogenic shift in VSMCs. Int J Cardiol 2019;297:94-103.

50. Trajkovic K, Hsu C, Chiantia S, et al. Ceramide triggers budding of exosome vesicles into multivesicular Endosomes. Science 2008;319:1244-7.

51. Voelkl J, Lang F, Eckardt KU, et al. Signaling pathways involved in vascular smooth muscle cell calcification during hyperphosphatemia. Cell Mol Life Sci 2019;76:2077-91.

52. Icer MA, Yildiran H. Effects of fetuin-A with diverse functions and multiple mechanisms on human health. Clin Biochem 2021;88:1-10.

53. Anderson HC, Mulhall D, Garimella R. Role of extracellular membrane vesicles in the pathogenesis of various diseases, including cancer, renal diseases, atherosclerosis, and arthritis. Lab Invest 2010;90:1549-57.

54. Chen YD, Fang YT, Cheng YL, et al. Exophagy of annexin A2 via RAB11, RAB8A and RAB27A in IFN-gammastimulated lung epithelial cells. Sci Rep 2017;7:5676.

55. Cui L, Houston DA, Farquharson C, et al. Characterisation of matrix vesicles in skeletal and soft tissue mineralisation. Bone 2016;87:147-58.

56. Lanzer P, Boehm M, Sorribas V, et al. Medial vascular calcification revisited: review and perspectives. Eur Heart J 2014;35:1515-25.

57. Moe SM, Duan D, Doehle BP, et al. Uremia induces the osteoblast differentiation factor Cbfa1 in human blood vessels. Kidney Int 2003;63:1003-11.

58. Tyson KL, Reynolds JL, McNair R, et al. Osteo/ chondrocytic transcription factors and their target genes exhibit distinct patterns of expression in human arterial calcification. Arterioscler Thromb Vasc Biol 2003;23:489-94.

59. Allahverdian S, Chaabane C, Boukais K, et al. Smooth muscle cell fate and plasticity in atherosclerosis. Cardiovasc Res 2018;114:540-50.

60. Kelly-Arnold A, Maldonado N, Laudier D, et al. Revised microcalcification hypothesis for fibrous cap rupture in human coronary arteries. Proc Natl Acad Sci U S A 2013;110:10741-6.

61. Li S, Zhan JK, Wang YJ, et al. Exosomes from hyperglycemia-stimulated vascular endothelial cells contain versican that regulate calcification/senescence in vascular smooth muscle cells. Cell Biosci 2019;9:1.

62. Maegdefessel L, Rayner KJ, Leeper NJ. MicroRNA Regulation of Vascular Smooth Muscle Function and Phenotype Early Career Committee Contribution. Arterioscler Thromb Vasc Biol 2015;35:2-6.
63. Goettsch C, Hutcheson JD, Aikawa M, et al. Sortilin mediates vascular calcification via its recruitment into extracellular vesicles. J Clin Invest 2016;126:1323-36.

64. Nik AB, Hutcheson JD, Aikawa E. Extracellular Vesicles As Mediators of Cardiovascular Calcification. Front Cardiovasc Med 2017;4:78.

65. Whitehead M, Ahmad S, Shanahan C. Role of vascular smooth muscle cell derived-exosomes in age-related vascular amyloidosis. Heart 2018;104:A96.

66. Bardeesi ASA, Gao JW, Zhang K, et al. A novel role of cellular interactions in vascular calcification. J Transl Med 2017;15:95.

67. Yu X, Odenthal M, Fries JWU. Exosomes as miRNA Carriers: Formation-Function-Future. Int J Mol Sci 2016;17:2028.

68. Mackenzie NCW, Staines KA, Zhu D, et al. miRNA-221 and miRNA-222 synergistically function to promote vascular calcification. Cell Biochem Funct 2014;32:209-16.

69. Gui T, Zhou G, Sun Y, et al. MicroRNAs that target Ca2+ transporters are involved in vascular smooth muscle cell calcification. Lab Invest 2012;92:1250-9.

70. Eijken M, Swagemakers S, Koedam M, et al. The activin A-follistatin system: potent regulator of human extracellular matrix mineralization. FASEB J 2007;21:2949-60.

71. Icli B, Wara AKM, Moslehi J, et al. MicroRNA-26a Regulates Pathological and Physiological Angiogenesis by Targeting BMP/SMAD1 Signaling. Circ Res 2013;113:1231-41.

72. Balderman JAF, Lee HY, Mahoney CE, et al. Bone Morphogenetic Protein-2 Decreases MicroRNA-30b and MicroRNA-30c to Promote Vascular Smooth Muscle Cell Calcification. J Am Heart Assoc 2012;1:e003905.

73. Li Z, Hassan MQ, Volinia S, et al. A microRNA signature for a BMP2-induced osteoblast lineage commitment program. Proc Natl Acad Sci U S A 2008;105:13906-11.

74. Cao Y, Zhou ZC, de Crombrugghe B, et al. Osterix, a transcription factor for osteoblast differentiation, mediates antitumor activity in murine osteosarcoma. Cancer Res 2005;65:1124-8.

75. Goettsch C, Rauner M, Pacyna N, et al. miR-125b Regulates Calcification of Vascular Smooth Muscle Cells. Am J Pathol 2011;179:1594-600.

76. Cui RR, Li SJ, Liu LJ, et al. MicroRNA-204 regulates vascular smooth muscle cell calcification in vitro and in vivo. Cardiovasc Res 2012;96:320-9.

77. Huang X, Liang M, Dittmar R, et al. Extracellular MicroRNAs in Urologic Malignancies: Chances and 
Challenges. Int J Mol Sci 2013;14:14785-99.

78. Skog J, Würdinger T, van Rijn S, et al. Glioblastoma microvesicles transport RNA and proteins that promote tumour growth and provide diagnostic biomarkers. Nat Cell Biol 2008;10:1470-6.

79. Yang VK, Loughran KA, Meola DM, et al. Circulating exosome microRNA associated with heart failure secondary to myxomatous mitral valve disease in a naturally occurring canine model. J Extracell Vesicles 2017;6:1350088.

80. Cui Y, Luan J, Li H, et al. Exosomes derived from mineralizing osteoblasts promote ST2 cell osteogenic differentiation by alteration of microRNA expression. Febs Letters 2016;590:185-92.

81. Guo Y, Bao S, Guo W, et al. Bone marrow mesenchymal stem cell-derived exosomes alleviate high phosphorusinduced vascular smooth muscle cells calcification by modifying microRNA profiles. Funct Integr Genomics 2019;19:633-43.

82. Rangrez AY, M'Baya-Moutoula E, Metzinger-Le Meuth $\mathrm{V}$, et al. Inorganic Phosphate Accelerates the Migration of Vascular Smooth Muscle Cells: Evidence for the Involvement of miR-223. PLoS One 2012;7:e47807.

83. Ni YQ, Lin X, Zhan JK, et al. Roles and Functions of Exosomal Non-coding RNAs in Vascular Aging. Aging Dis 2020;11:164-78.

84. Yang X, Dong M, Wen H, et al. MiR-26a contributes to the PDGF-BB-induced phenotypic switch of vascular smooth muscle cells by suppressing Smad1. Oncotarget 2017;8:75844-53

85. Zhao Y, Li Y, Luo P, et al. XBP1 splicing triggers miR-150 transfer from smooth muscle cells to endothelial cells via extracellular vesicles. Sci Rep 2016;6:28627.

86. Lombardo G, Dentelli P, Togliatto G, et al. Activated Stat5 trafficking Via Endothelial Cell-derived Extracellular Vesicles Controls IL-3 Pro-angiogenic Paracrine Action. Sci Rep 2016;6:25689.

87. Hergenreider E, Heydt S, Treguer K, et al. Atheroprotective communication between endothelial cells and smooth muscle cells through miRNAs. Nature Cell Biology 2012;14:249-56.

88. Lin X, Li S, Wang YJ, et al. Exosomal Notch3 from high glucose-stimulated endothelial cells regulates vascular smooth muscle cells calcification/aging. Life Sci 2019;232:116582.

89. Cerezo-Magana M, Bang-Rudenstam A, Belting M. The pleiotropic role of proteoglycans in extracellular vesicle mediated communication in the tumor microenvironment. Semin Cancer Biol 2020;62:99-107.
90. Christianson HC, Svensson KJ, van Kuppevelt TH, et al. Cancer cell exosomes depend on cell-surface heparan sulfate proteoglycans for their internalization and functional activity. Proc Natl Acad Sci U S A 2013;110:17380-5.

91. Mattoscio D, Casadio C, Miccolo C, et al. Autophagy regulates UBC9 levels during viral mediated tumorigenesis. Plos Pathogens 2017;13:e1006262.

92. Blanc L, Vidal M. New insights into the function of Rab GTPases in the context of exosomal secretion. Small GTPases 2018;9:95-106.

93. Dai XY, Zhao MM, Cai Y, et al. Phosphate-induced autophagy counteracts vascular calcification by reducing matrix vesicle release. Kidney Int 2013;83:1042-51.

94. Bartsch H, Nair J. Chronic inflammation and oxidative stress in the genesis and perpetuation of cancer: role of lipid peroxidation, DNA damage, and repair. Langenbecks Arch Surg 2006;391:499-510.

95. Gamboa JL, Billings FT, Bojanowski MT, et al. Mitochondrial dysfunction and oxidative stress in patients with chronic kidney disease. Physiol Rep 2016;4:e12780.

96. Liakopoulos V, Roumeliotis S, Gorny X, et al. Oxidative Stress in Hemodialysis Patients: A Review of the Literature. Oxid Med Cell Longev 2017;2017:3081856.

97. Wei Q, Ren XM, Jiang YB, et al. Advanced glycation end products accelerate rat vascular calcification through RAGE/oxidative stress. BMC Cardiovasc Disord 2013;13:13.

98. Wei R, Enaka M, Muragaki Y. Activation of KEAP1/ NRF2/P62 signaling alleviates high phosphate-induced calcification of vascular smooth muscle cells by suppressing reactive oxygen species production. Sci Rep 2019;9:10366.

99. Patel GK, Khan MA, Bhardwaj A, et al. Exosomes confer chemoresistance to pancreatic cancer cells by promoting ROS detoxification and miR-155-mediated suppression of key gemcitabine-metabolising enzyme, DCK. Br J Cancer 2017;116:609-19.

100.Liu J, Xiao X, Shen Y, et al. MicroRNA-32 promotes calcification in vascular smooth muscle cells: Implications as a novel marker for coronary artery calcification. PLoS One 2017;12:e0174138.

101. Hagiwara S, McClelland A, Kantharidis P. MicroRNA in Diabetic Nephropathy: Renin Angiotensin, AGE/ RAGE, and Oxidative Stress Pathway. J Diabetes Res 2013;2013:173783.

102. Shi S, Yu L, Chiu C, et al. Podocyte-Selective Deletion of Dicer Induces Proteinuria and Glomerulosclerosis. J Am Soc Nephrol 2008;19:2159-69. 
103. Gong M, Yu B, Wang JC, et al. Mesenchymal stem cells release exosomes that transfer miRNAs to endothelial cells and promote angiogenesis. Oncotarget 2017;8:45200-12.

104. Wang Y, Ma WQ, Zhu Y, et al. Exosomes Derived From Mesenchymal Stromal Cells Pretreated With Advanced Glycation End Product-Bovine Serum Albumin Inhibit Calcification of Vascular Smooth Muscle Cells. Front Endocrinol (Lausanne) 2018;9:524.

Cite this article as: Qin Z, Liao R, Xiong Y, Jiang L, Li J, Wang L, Han M, Sun S, Geng J, Yang Q, Zhang Z, Li Y, Du H, $\mathrm{Su}$ B. A narrative review of exosomes in vascular calcification. Ann Transl Med 2021;9(7):579. doi: 10.21037/atm-20-7355
105.Nakano-Kurimoto R, Ikeda K, Uraoka M, et al. Replicative senescence of vascular smooth muscle cells enhances the calcification through initiating the osteoblastic transition. Am J Physiol Heart Circ Physiol 2009;297:H1673-84.

106.Zhan JK, Wang YJ, Li S, et al. AMPK/TSC2/mTOR pathway regulates replicative senescence of human vascular smooth muscle cells. Exp Ther Med 2018;16:4853-8. 